\title{
Silencing of the long noncoding RNA LINC01132 alleviates the oncogenicity of epithelial ovarian cancer by regulating the microRNA-431-5p/SOX9 axis
}

\author{
WEI ZHU ${ }^{1}$, XIANGMING XIAO ${ }^{2}$ and JINQIN CHEN ${ }^{1}$ \\ Departments of ${ }^{1}$ Gynaecology and Obstetrics and ${ }^{2}$ General Surgery, Weifang People's Hospital, \\ Weifang, Shandong 261401, P.R. China
}

Received February 5, 2021; Accepted May 6, 2021

DOI: $10.3892 / \mathrm{ijmm} .2021 .4984$

\begin{abstract}
To date, the role of lncRNA long intergenic non-protein-coding RNA 1132 (LINC01132) expression in epithelial ovarian cancer (EOC) has not been explored. Thus, LINC01132 expression in EOC was assessed and the regulatory activity of LINC01132 on the malignant behaviours of EOC cells was investigated. Additionally, the molecular events that occurred downstream of LINC01132 in EOC cells were also revealed. In the present study, LINC01132 expression in EOC was verified by employing RT-qPCR. The effects of LINC01132 on the aggressive behaviours of EOC cells were revealed utilizing multiple functional experiments. The targeting interaction among LINC01132, microRNA-431-5p (miR-431-5p) and SRY-box 9 (SOX9) was demonstrated by RNA immunoprecipitation and luciferase reporter assay. Herein, LINC01132 was overexpressed in EOC and was significantly associated with poor patient prognosis. Functionally, cell experiments revealed that LINC01132 depletion produced cancer-suppressive effects in EOC cells and regulated cell proliferation, migration, invasion and apoptosis in vitro. Additionally, the loss of LINC01132 attenuated tumour growth in vivo. Mechanistically, LINC01132 acted as a competing endogenous RNA by sequestering miR-431-5p and consequently overexpressing SOX9 in EOC cells, forming a LINC01132/miR-431-5p/SOX9 axis. In rescue experiments, miR-431-5p inhibition or SOX9 reintroduction eliminated the anti-tumour effects of LINC01132 silencing on the pathological behaviours of EOC cells. Generally, LINC01132 exhibited oncogenic activities in EOC cells by regulating the outcome of the miR-431-5p/SOX9 axis, providing an effective target for EOC diagnosis, therapy and prognosis evaluation.
\end{abstract}

Correspondence to: Professor Jinqin Chen, Department of Gynaecology and Obstetrics, Weifang People's Hospital, 151 Guangwen Road, Weifang, Shandong 261401, P.R. China E-mail: chenjinqin_ovarian@126.com

Key words: long noncoding RNA, ceRNA network, miRNA sponge, LINC01132

\section{Introduction}

Ovarian cancer, a lethal gynaecological tumour, is the fifth most common cause of tumour-associated mortalities among females (1). Ovarian cancer ranks first among gynaecological malignancies in terms of mortality, with a 5-year survival rate of $<35 \%$ (2). Every year, approximately 150,000 patients succumb to ovarian cancer, and this number is increasing annually (3). Epithelial ovarian cancer (EOC) is the major type of ovarian cancer, accounting for approximately $85-90 \%$ of all ovarian cancer cases and is associated with poor clinical outcomes (4). Currently, EOC is primarily treated with surgical resection along with chemotherapy, radiotherapy and immunological therapy; however, although numerous patients with EOC experience favourable therapeutic effects, EOC patients diagnosed at an advanced stage have poor prognosis after treatment with first-line therapies $(5,6)$. Furthermore, due to its subtle symptoms and signs, EOC is usually diagnosed in later stages, with pelvic dissemination or distant metastasis already present; thus, patients miss the optimal timing for surgical intervention and have reduced survival (7). Therefore, understanding the mechanisms of EOC pathogenesis is urgently required to identify promising targets for tumour diagnosis and management.

Long noncoding RNAs (lncRNAs) are a heterogeneous class of RNA transcripts consisting of over 200 nucleotides (8). Although lncRNAs lack the ability to code proteins, they participate in controlling gene expression (9). Functionally, IncRNAs have been confirmed to be crucial modulators of diverse physiological and pathological processes, especially during tumorigenesis and cancer progression (10). Increasing evidence has indicated that an imbalance in lncRNA expression is closely associated with EOC progression (11-13). The dysregulation of IncRNAs has been clearly demonstrated to exert cancer-inhibiting or cancer-facilitating effects and to contribute to the aggressive properties of EOC (14-16).

MicroRNAs (miRNAs) belong to a group of small, nonprotein-coding RNA transcripts that are known to negatively regulate gene expression by base pairing with target genes and consequently causing mRNA degradation or translation suppression (17). Convincing evidence has revealed the important regulatory activities of miRNAs in EOC oncogenesis 
through their pro- or anti-tumourigenic effects (18-20). Recently, the competing endogenous RNA (ceRNA) theory was proposed to explain the mechanisms of IncRNA action; lncRNAs directly sequester miRNAs and thereby modulate gene expression by weakening the miRNA-mediated suppression of gene expression $(21,22)$. Thus, exploring cancer-related lncRNAs and miRNAs, as well as the ceRNA theory, could be a feasible approach for understanding the mechanisms underlying EOC progression.

Long intergenic non-protein-coding RNA 1132 (LINC01132) expression in EOC and the underlying mechanisms have not yet been explored. Thus, the expression level of LINC01132 in EOC was detected and its correlation with patient prognosis was examined. Next, functional experiments were performed to assess the effects of LINC01132 on the aggressive behaviours of EOC cells. Furthermore, the mechanisms that occurred downstream of LINC01132 in EOC cells were elucidated.

\section{Materials and methods}

Tissue specimens and cell lines. The collection and use of human tissues was approved (approval no. EC-WFPH.20150106) by the Ethics Committee of Weifang People's Hospital (Weifang, China). After obtaining the written informed consent, 51 pairs of EOC tissues and adjacent normal tissues were obtained from patients (age range, 46-69 years) at Weifang People's Hospital from February 2015 to January 2016. The inclusion criteria included: i) Diagnosed with EOC and agreed to take part in this research; and ii) had not received chemotherapy or radiotherapy prior to surgery. Patients who had other types of human cancer or had been treated with chemotherapy or radiotherapy were excluded. ES-2, an EOC cell line, was obtained from American Type Culture Collection (ATCC) and was cultured in McCoy's 5A Medium that was supplemented with $10 \%$ foetal bovine serum (FBS) (Gibco; Thermo Fisher Scientific, Inc.). The 3 EOC cell lines, namely, OVCAR3, CAOV-3 and SK-OV-3 were obtained from the National Collection of Authenticated Cell Cultures (Shanghai, China). The culture conditions for the SK-OV-3 cell line were the same as those for the ES-2 cell line. CAOV-3 and OVCAR 3 cells were maintained in 10\% FBS-supplemented DMEM and 20\% FBS-supplemented RPMI-1640 medium (both from Gibco; Thermo Fisher Scientific, Inc.), respectively. Bovine insulin $(0.01 \mathrm{mg} / \mathrm{ml}$; Gibco; Thermo Fisher Scientific, Inc.) was also used for OVCAR3 cells. The human ovarian surface epithelial cell line (OSE) was grown in ovarian epithelial cell medium (both from ScienCell Research Laboratories, Inc.). All cell lines aforementioned were cultured at $37^{\circ} \mathrm{C}$ in an incubator containing a humidified (100\%) atmosphere with $5 \% \mathrm{CO}_{2}$.

Transfection experiment. To overexpress miR-431-5p and SOX9, transfection of the miR-431-5p mimic (100 pmol; Shanghai GenePharma Co., Ltd.) and pcDNA3.1-SOX9 (4 $\mu \mathrm{g}$; Sangon Biotech Co., Ltd.) was conducted with Lipofectamine ${ }^{\circledR} 2000$ reagent (Invitrogen; Thermo Fisher Scientific, Inc.), and the miRNA mimic control (100 pmol; miR-NC; Shanghai GenePharma Co., Ltd.) and pcDNA3.1 $(4 \mu \mathrm{g}$;) were used as controls. All transfections were performed at room temperature. The transfection duration was $8 \mathrm{~h}$, after which the culture medium was replaced with fresh culture medium.

Small interfering RNAs (siRNAs) targeting LINC01132 (si-LINC01132; 100 pmol) and the miR-431-5p inhibitor were prepared by Shanghai GenePharma Co., Ltd. and used to knock down LINC01132 and miR-431-5p. The negative control (NC) siRNA (si-NC; $100 \mathrm{pmol}$ ) and NC inhibitor functioned as the controls for si-LINC01132 and the miR-431-5p inhibitor, respectively. The miR-431-5p mimic sequence was 5'-ACGUACUGCCGGACGUUCUGU-3' and the miR-NC sequence was 5'-UUGUACUACACAAAAGUACUG-3'. The miR-431-5p inhibitor sequence was 5'-UGCAUGACG GCCUGCAAGACA-3' and the NC inhibitor sequence was 5'-ACUACUGAGUGACAGUAGA-3'. The si-LINC01132\#1 sequence was 5'-AGGAGATAAAAATTTTAAATTAC-3'; the si-LINC01132\#2 sequence was 5'-TTCTGTTTTTTGTTT TTTTAAGA-3'; and the si-NC sequence was 5'-CACGAT AAGACAATGTATTT-3'.

RNA extraction and reverse transcription-quantitative PCR $(R T-q P C R)$. TRIzol ${ }^{\circledR}$ reagent (Invitrogen; Thermo Fisher Scientific, Inc.) was employed for total RNA isolation from tissues, cells or tumor xenografts. The RNA was quantified with a NanoDrop ${ }^{\mathrm{TM}} 2000$ spectrophotometer (Invitrogen; Thermo Fisher Scientific, Inc.) at a 260 -nm wavelength. For the determination of miRNA expression, first-strand cDNA was synthesized by applying Mir-X miRNA First-Strand Synthesis kit (Takara Biotechnology Co., Ltd.), according to the manufacturer's instructions. Next, PCR quantification was implemented utilizing Mir-X miRNA RT-qPCR TB Green ${ }^{\circledR}$ kit (Takara Biotechnology Co., Ltd.) in accordance with the manufacturer's instructions. U6 small nuclear RNA acted as the internal reference for miRNAs.

For the determination of LINC01132 and SOX9 levels, PrimeScript $^{\mathrm{TM}}$ RT reagent kit with gDNA Eraser and TB Green ${ }^{\circledR}$ Premix Ex Taq ${ }^{\mathrm{TM}}$ II (Takara Biotechnology Co., Ltd.) were, respectively, adopted for reverse transcription and PCR amplification. Both kits were used according to the manufacturer's instructions. The thermocycling conditions for PCR amplification were as follows: Initial hold at $95^{\circ} \mathrm{C}$ for $30 \mathrm{sec}$; 40 cycles of amplification at $95^{\circ} \mathrm{C}$ for $3 \mathrm{sec}$, and annealing for $30 \mathrm{sec}$ at $60^{\circ} \mathrm{C}$ and extension at $72^{\circ} \mathrm{C}$ for $30 \mathrm{sec}$. GAPDH acted as the internal reference for LINC01132 and SOX9 in this assay. The $2^{-\Delta \Delta \mathrm{Cq}}$ (23) quantification method was used to calculate the relative mRNA expression levels. The primers were designed as follows: LINC01132 forward, 5'-CGGAAGCAG GGACTGCTATT-3' and reverse, 5'-TCCTGGTGGCTCTGT CCTCTC-3'; SOX9 forward, 5'-GACGTCATCTCCAACATC GAGACCT-3' and reverse, 5'-GCTGCCCGTGTAGGTGAC CT-3'; GAPDH forward, 5'-CGGAGTCAACGGATTTGG TCGTAT-3' and reverse, 5'-AGCCTTCTCCATGGTGGTGAA GAC-3'; U6 forward, 5'-CTCGCTTCGGCAGCACA-3' and reverse, 5'-AACGCTTCACGAATTTGCGT-3; miR-125b-5p forward, 5'-TCGGCAGGUCCCUGAGACC-3' and reverse, 5'-CACTCAACTGGTGTCGTGGA-3'; miR-134-3p forward, 5'-TCGGCAGGAACCACUGAUC-3' and reverse, 5'-CAC TCAACTGGTGTCGTGGA-3'; miR-199a-3p forward, 5'-TCG GCAGGAUUGGUUACACG-3' and reverse, 5'-CACTCA ACTGGTGTCGTGGA-3'; miR-199b-3p forward, 5'-TCG GCAGGAUUGGUUACACG-3' and reverse, 5'-CACTCA 
ACTGGTGTCGTGGA-3'; miR-431-5p forward, 5'-TCGGCA GGUGUCUUGCAGG-3' and reverse, 5'-CACTCAACTGGT GTCGTGGA-3'.

Cell Counting Kit-8 (CCK-8) assay. EOC cell proliferation was assessed using a CCK-8 assay (Dojindo Molecular Technologies, Inc.). In detail, every well of 96 -well plates was seeded with 2,000 transfected cells that were resuspended in $100 \mu \mathrm{l}$ of complete culture medium. At $0,1,2$, and 3 days after adherence to the wells, $10 \mu 1$ of CCK- 8 solution was added, and the cells were cultured for an additional $2 \mathrm{~h}$ under the conditions aforementioned. The absorbance was monitored at a wavelength of $450 \mathrm{~nm}$ via a microplate reader.

Flow cytometric analysis. Transfected cells $\left(1 \times 10^{6}\right)$ were digested with trypsin (Gibco; Thermo Fisher Scientific, Inc.), rinsed with ice-cooled phosphate-buffered saline and centrifuged at room temperature at $1,000 \mathrm{x}$ g for $5 \mathrm{~min}$. The apoptosis of these cells was analysed by employing an Annexin V-fluorescein isothiocyanate (FITC) apoptosis detection kit (Beyotime Institute of Biotechnology) in accordance with the manufacturer's instructions. Briefly, the cells were resuspended in $195 \mu \mathrm{l}$ of Annexin V-FITC binding buffer and cultured with $5 \mu \mathrm{l}$ of Annexin V-FITC and $10 \mu \mathrm{l}$ of PI at $25^{\circ} \mathrm{C}$. The incubation was performed in the dark and continued for $20 \mathrm{~min}$. All samples were analysed using a flow cytometer (FACSCalibur; BD Biosciences). CellQuest software (version 2.9; BD Biosciences) was applied for data analysis.

Cell migration and invasion assays. The EOC cell migration and invasion abilities were evaluated using the Transwell method ( $8-\mu \mathrm{m}$ pores; BD Biosciences). In the migration assay, the upper chambers were filled with $200 \mu 1$ of serum-free RPMI-1640 medium (for OVCAR3) or DMEM medium (for CAOV-3) containing $5 \times 10^{4}$ cells. By serving as a chemoattractant, a volume of $20 \%$ FBS-supplemented $600 \mu 1$ of culture medium was added to the lower compartments. After 1 day of culture, the non-migrated cells were wiped off by applying a cotton swab, and the migrated cells that crossed the pores were fixed using $100 \%$ methanol at room temperature for $30 \mathrm{~min}$ and stained using $0.1 \%$ crystal violet. In the invasion assay, Matrigel-coated Transwell chambers (BD Biosciences) were utilized, and the experimental procedures were the same as those of the migration assay. The precoating with Matrigel was conducted at $37^{\circ} \mathrm{C}$ for $2 \mathrm{~h}$. The number of stained cells in 5 random fields of view was counted with a light microscope (magnification, x200).

Tumor xenograft model. A second-generation lentiviral system was applied for the production of lentiviruses. LINC01132 short hairpin RNA (shRNA; sh-LINC01132) and NC shRNA (sh-NC) were prepared by Shanghai GenePharma Co., Ltd. After these molecules were inserted into a pLKO.1 lentiviral vector (Addgene Inc.), the vectors were transfected into 293T cells (National Collection of Authenticated Cell Cultures) with psPAX2 and pMD2.G. A total of $30 \mu \mathrm{g}$ of plasmids were used for lentivirus packaging, and the ratio of lentiviral plasmid: pLKO.1: psPAX2: pMD2.G was 2:1:1. Approximately $48 \mathrm{~h}$ after transfection, the lentiviruses were collected and used to infect CAOV-3 cells (MOI=5). After 3 days, fresh complete culture medium containing puromycin was used to further incubate the CAOV-3 cells, yielding cells stably overexpressing sh-LINC01132 or sh-NC. The concentrations of puromycin used for selection and maintenance were 2 and $0.3 \mu \mathrm{g} / \mathrm{ml}$, respectively.

All experimental steps involving animals were conducted with the approval from the Institutional Animal Care and Use Committee of Weifang People's Hospital (Weifang, China). Four-week-old female BALB/c nude mice $(n=6 ; 20 \mathrm{~g})$ were acquired from Shanghai SLAC Laboratory Animal Co. Ltd. (Shanghai, China). The mice were housed under specific pathogen-free conditions at $25^{\circ} \mathrm{C}$ and $50 \%$ humidity, with a 10:14 light/dark cycle and ad libitum access to food and water. Stably transfected cells $\left(2 \times 10^{6}\right)$ were harvested and subcutaneously injected into the flanks of nude mice. One week later, the sizes of the subcutaneous tumours were recorded every 4 days, and their volumes were calculated with the formula: Volume $\left(\mathrm{mm}^{3}\right)=1 / 2 \mathrm{x}$ length $\mathrm{x}$ width ${ }^{2}$. The mice were euthanized by cervical dislocation on day 31 , and the subcutaneous tumours were harvested for subsequent use.

Bioinformatics prediction. The online software miRDB (http://mirdb.org/) was adopted to identify the downstream target of LINC01132. The direct target of miR-431-5p was predicted utilizing TargetScan (version 7.2; http://www. targetscan.org) and miRDB.

TCGA and GTEx databases. TCGA (https://portal. gdc.cancer.gov/) and GTEx (https://www.genome. gov/Funded-Programs-Projects/Genotype-Tissue-ExpressionProject) were utilized to analyse LINC01132 expression in ovarian cancer.

Subcellular fractionation assay. The lncLocator (http://www. csbio.sjtu.edu.cn/bioinf/lncLocator/) was utilized to predict subcellular distribution of LINC01132 in human cells. The prediction was then verified by a Cytoplasmic and Nuclear RNA Purification kit (Norgen Biotek Corp.), which was used according to the manufacturer's instructions. EOC cells were processed with $200 \mu 1$ ice-cold cell fractionation buffer for 5 min to separate the cytoplasm and nucleus. The relative expression of LINC01132 in both fractions was measured by RT-qPCR.

Luciferase reporter assay. LINC01132 and the SOX9 3'-UTR fragments harbouring wild-type (wt) miR-431-5p-binding sequences were amplified and cloned into the downstream region of the psiCHECK $^{\mathrm{TM}}-2$ vector (Promega Corporation). The luciferase reporter vectors were labelled LINC01132-wt and SOX9-wt. The corresponding mutant (mut) luciferase reporter vectors, namely, psiCHECK ${ }^{\mathrm{TM}}$-2-LINC01132-mut and psiCHECK ${ }^{\mathrm{TM}}$-2-SOX9-mut, were generated in the same manner. For the reporter assay, EOC cells were inoculated into 24-well plates with a density of $1.5 \times 10^{5}$ cells /well, and incubated at $37^{\circ} \mathrm{C}$ overnight. Cells were transfected with miR-431-5p mimic or miR-NC alongside wt or mut reporter vectors utilizing Lipofectamine ${ }^{\circledR} 2000$ reagent (Invitrogen; Thermo Fisher Scientific, Inc.). The transfected cells were lysed at $48 \mathrm{~h}$ post-transfection with a Dual-Luciferase Reporter Assay System (Promega Corporation) for luciferase 
A

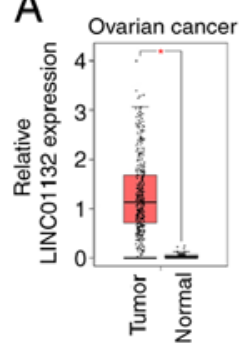

B

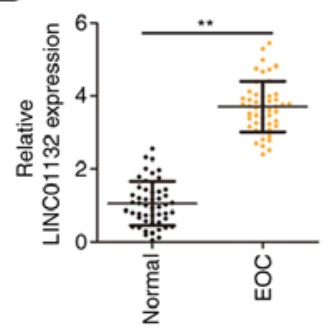

C

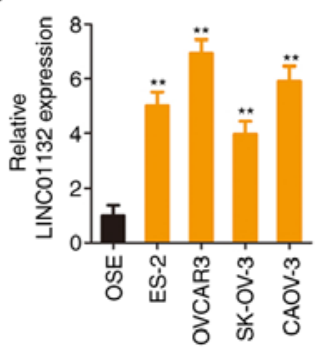

D

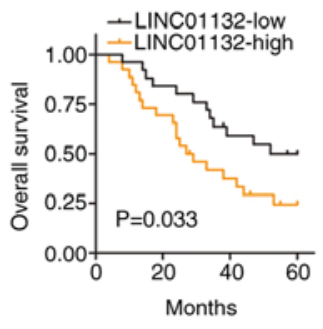

E

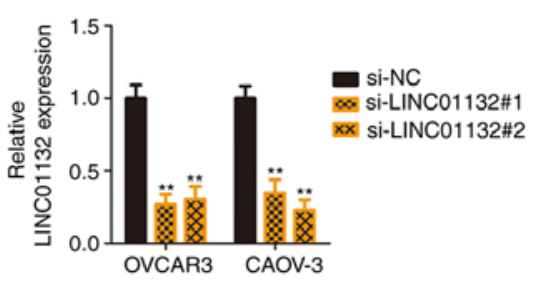

G

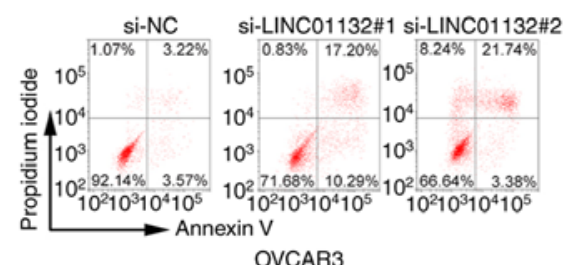

F

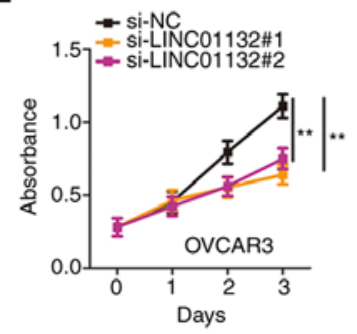

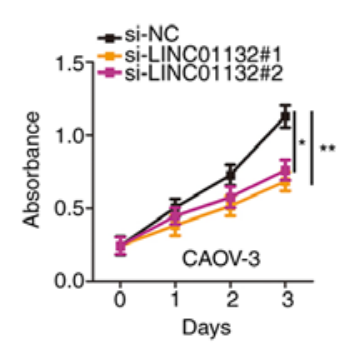
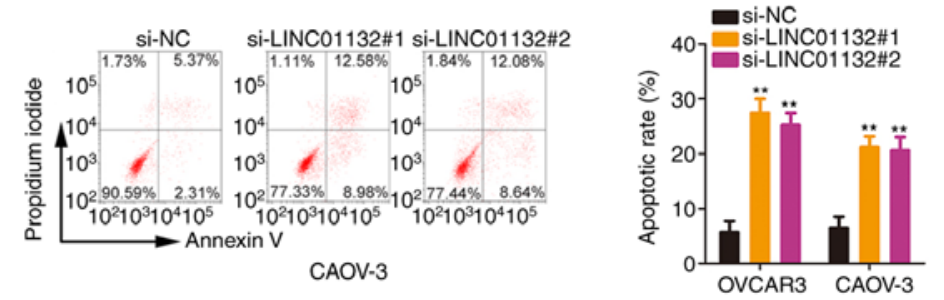

$\mathrm{H}$

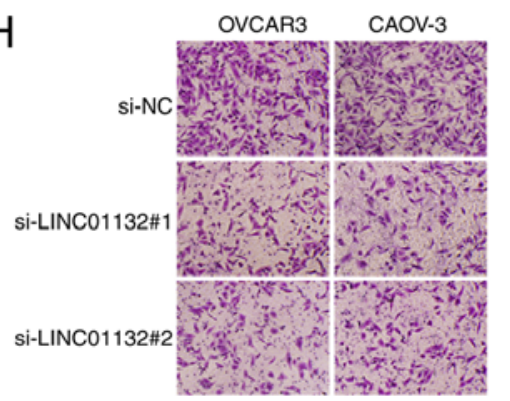

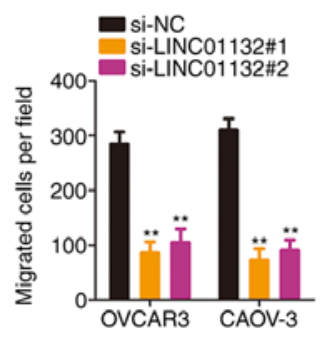

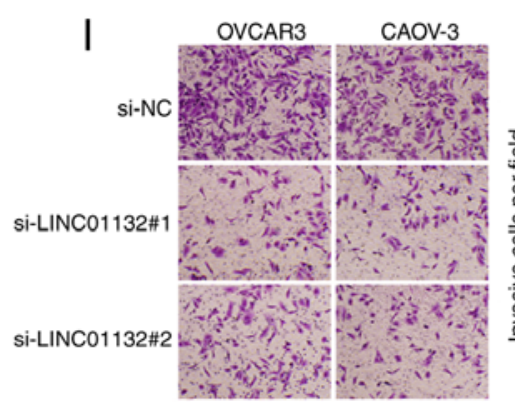

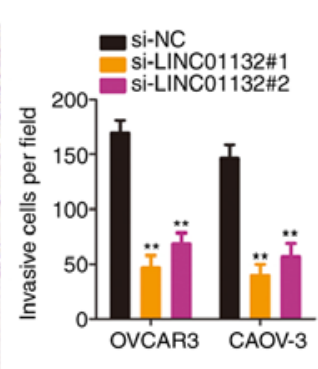

Figure 1. LINC01132 is overexpressed in EOC. (A) LINC01132 expression pattern in ovarian cancer was analysed via The Cancer Genome Atlas and Genotype-Tissue Expression databases. (B) LINC01132 expression in EOC tissues. (C) LINC01132 level in EOC cell lines. (D) The relationship between the expression level of LINC01132 and overall survival in patients with EOC was revealed. (E) The knockdown efficiency of si-LINC01132 was evaluated using reverse transcription-quantitative PCR. (F) The proliferative ability of EOC cells after LINC01132 silencing was analysed. (G) Flow cytometric analysis detected alterations in the apoptosis of EOC cells after si-LINC01132 transfection. (H and I) The migration and invasion abilities of EOC cells were determined after LINC01132 silencing. " $\mathrm{P}<0.05$ and ${ }^{* *} \mathrm{P}<0.01$. LINC01132, long intergenic non-protein coding RNA 1132; EOC, epithelial ovarian cancer; si-, small interfering; $\mathrm{NC}$, negative control.

activity determination. Renilla luciferase activity was used to normalize the firefly luciferase activity.

RNA immunoprecipitation (RIP). The EZ-Magna RIP ${ }^{\mathrm{TM}}$ RNA-Binding Protein Immunoprecipitation kit (cat. no. 17-701; EMD Millipore) was used in the assay, according to the manufacturer's instructions. After lysing the cells in complete RIP lysis buffer (EMD Millipore), the whole-cell lysates were collected and treated with magnetic beads coupled to an anti-Ago 2 antibody or normal mouse IgG (cat. no. 17-701; dilution, 1:5,000; both from EMD Millipore) at $4^{\circ} \mathrm{C}$ overnight. In addition, $10 \mu \mathrm{l}$ of whole-cell lysates was aliquoted for use as the input and served as the positive control. IgG acted as the negative control. Proteinase K (EMD Millipore) treatment (30 $\mathrm{min}$ at $55^{\circ} \mathrm{C}$ ) was applied to detach the proteins, and the immunoprecipitated RNAs were extracted. Finally, the relative enrichment of LINC01132, miR-431-5p and SOX9 in the immunoprecipitated RNAs was examined via RT-qPCR.

Protein extraction and western blotting. Total protein was extracted using RIPA lysis buffer (Beyotime Institute of Biotechnology). After total protein quantification using a bicinchoninic acid kit (Beyotime Institute of Biotechnology), equal amounts of proteins $(30 \mu \mathrm{g})$ were separated by $10 \%$ SDS-PAGE electrophoresis and transferred onto PVDF membranes. The membranes were blocked at room temperature in 5\% skimmed milk for $2 \mathrm{~h}$ and then incubated overnight at $4^{\circ} \mathrm{C}$ with primary antibodies against SOX9 (product code ab185966; 1:1,000 dilution; Abcam) or GAPDH (product code ab181602; 1:1,000 dilution; Abcam), followed by $1 \mathrm{~h}$ incubation 
A

IncLocator prediction result
\begin{tabular}{|l|l|}
\hline Subcellular locations & Score \\
\hline Cytoplasm & 0.791110810558 \\
\hline Nucleus & 0.0770687244398 \\
\hline Ribosome & 0.0334633372898 \\
\hline Cytosol & 0.0804614147429 \\
\hline Exosome & 0.0178957129698 \\
\hline \multicolumn{2}{|l|}{} \\
\cline { 2 - 2 } \\
\cline { 2 - 2 } \\
\cline { 2 - 2 } \\
\cline { 2 - 2 } & Prediction location \\
\hline
\end{tabular}

B

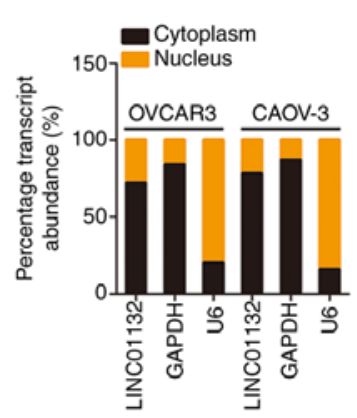

C
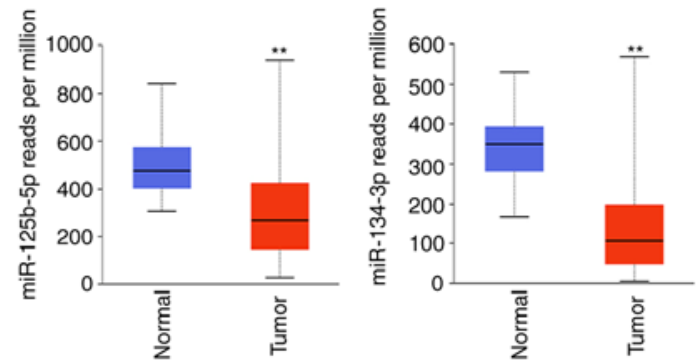

D

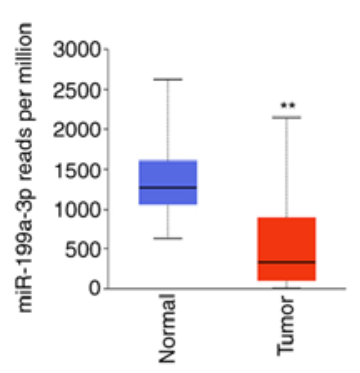

F

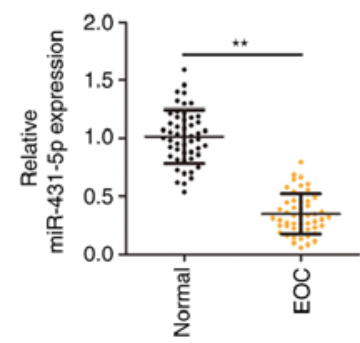

I

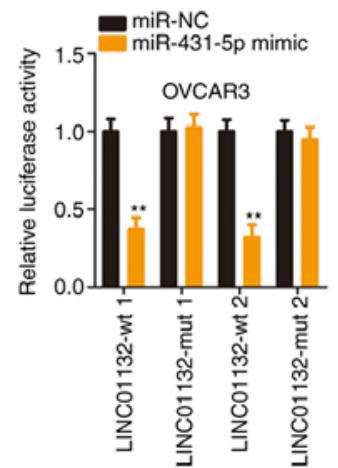

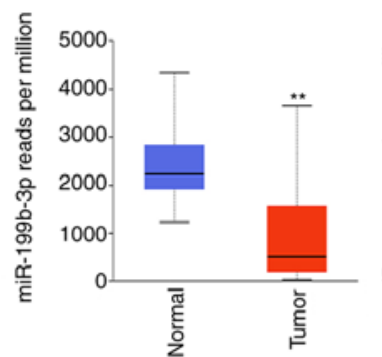

G
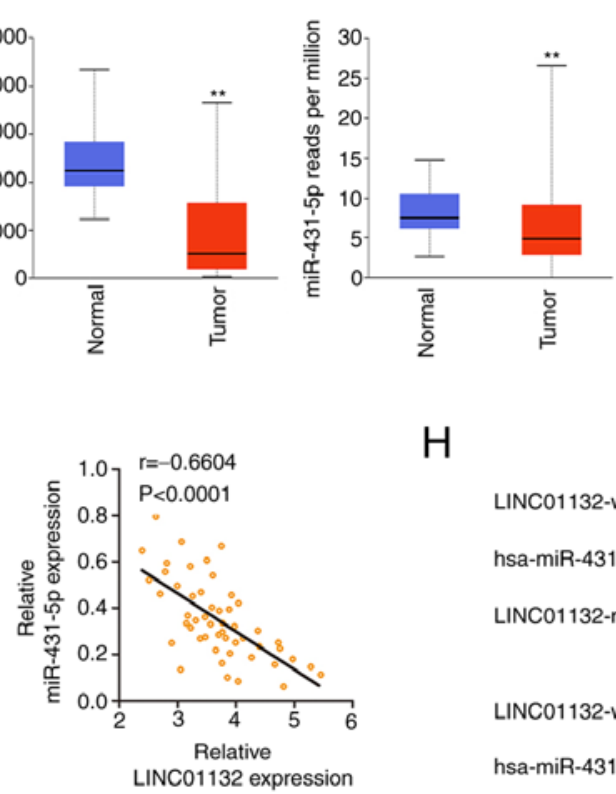

$\mathrm{H}$

LINC01132-wt

hsa-miR-431-5p

E

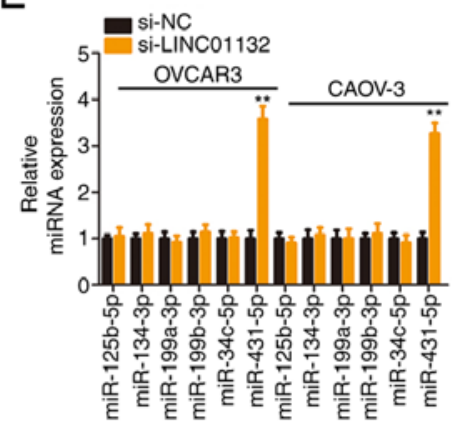

Site 1

LINC01132-wt $\quad 5^{\prime} \quad$...AUCUAGUUAGUGGGGU CAAGACA... $3^{\prime}$

hsa-miR-431-5p $3^{\prime}$

|||| $\mid$

ACGUACUGCCGGAC GUUCUGU 5

LINC01132-mut $\quad 5^{\prime}$...AUCUAGUUAGUGGGGU GUUCUGA... 3

Site 2

5' ...ACCAGAAACAAACAAGACC... 3'

LINC01132-mut
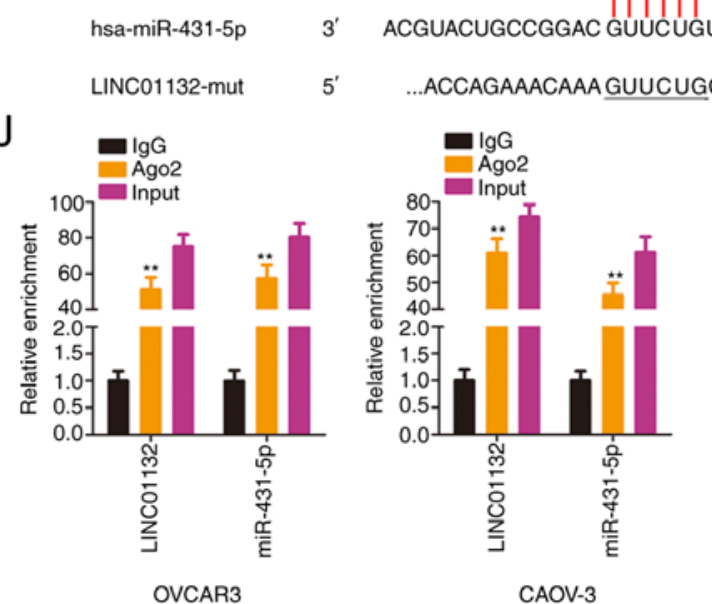

$5^{\prime} \quad$...ACCAGAAACAAAGUUCUGC... $3^{\prime}$
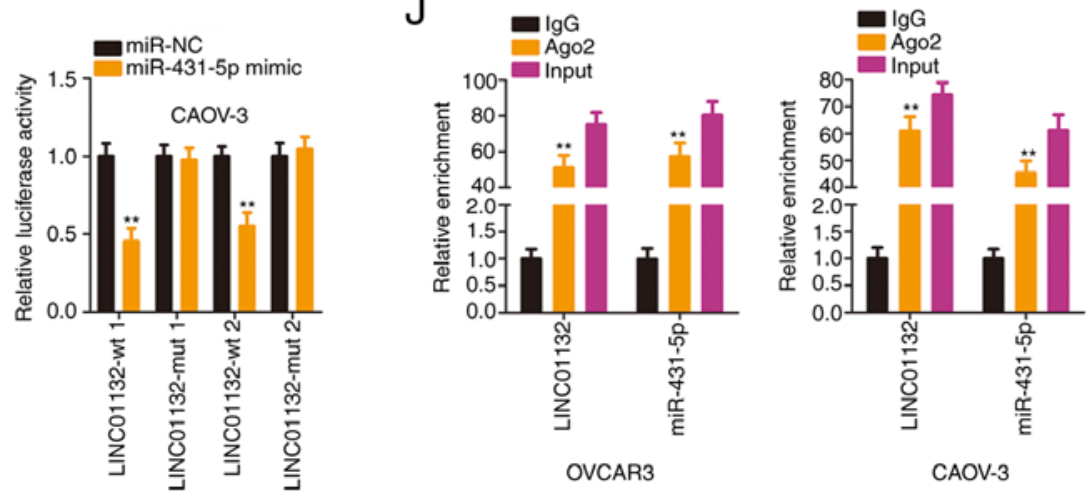

Figure 2. LINC01132 is capable of sponging miR-431-5p in EOC cells. (A) The distribution of LINC01132 predicted by lncLocator. (B) Localization of LINC01132 in EOC cells. (C and D) Expression of miR-125b-5p, miR-134-3p, miR-199a-3p, miR-199b-3p, and miR-431-5p in ovarian cancer was analysed via The Cancer Genome Atlas database. (E) Expression of the 5 candidates in EOC cells following LINC01132 silencing was measured by RT-qPCR. (F) miR-431-5p level in EOC tissues was examined by RT-qPCR. (G) The expression relationship between LINC01132 and miR-431-5p in EOC tissues. (H) The predicted binding sequences between LINC01132 and miR-431-5p were demonstrated. The sequences containing underscores were the site that was mutated in LINC01132. (I) Luciferase activity of wt or mut LINC01132 reporter plasmids was quantified in OVCAR3 and CAOV-3 cells in the presence of miR-431-5p mimic or miR-NC. (J) RNA immunoprecipitation demonstrated the co-existence of LINC01132 and miR-431-5p in immunoprecipitated RNA. ** P<0.01. LINC01132, long intergenic non-protein coding RNA 1132; miR, microRNA; EOC, epithelial ovarian cancer; RT-qPCR, reverse transcription-quantitative PCR; wt, wild-type; mut, mutant; si-, small interfering; NC, negative control.

at room temperature with an HRP-conjugated secondary antibody (product code ab205718; 1:5,000 dilution; Abcam). Ultimately, a BeyoECL plus detection kit (Beyotime Institute of Biotechnology) was used to visualize the protein bands. Quantity One software (version 4.62; Bio-Rad Laboratories, Inc.) was employed for densitometric analysis.
Statistical analysis. All experiments were repeated thrice and conducted in triplicate, and the experimental data are expressed as the mean \pm standard deviation. Comparison of the significance between two groups was conducted with both paired and unpaired Student's t-tests. The statistical significance among multiple groups was examined utilizing one-way 

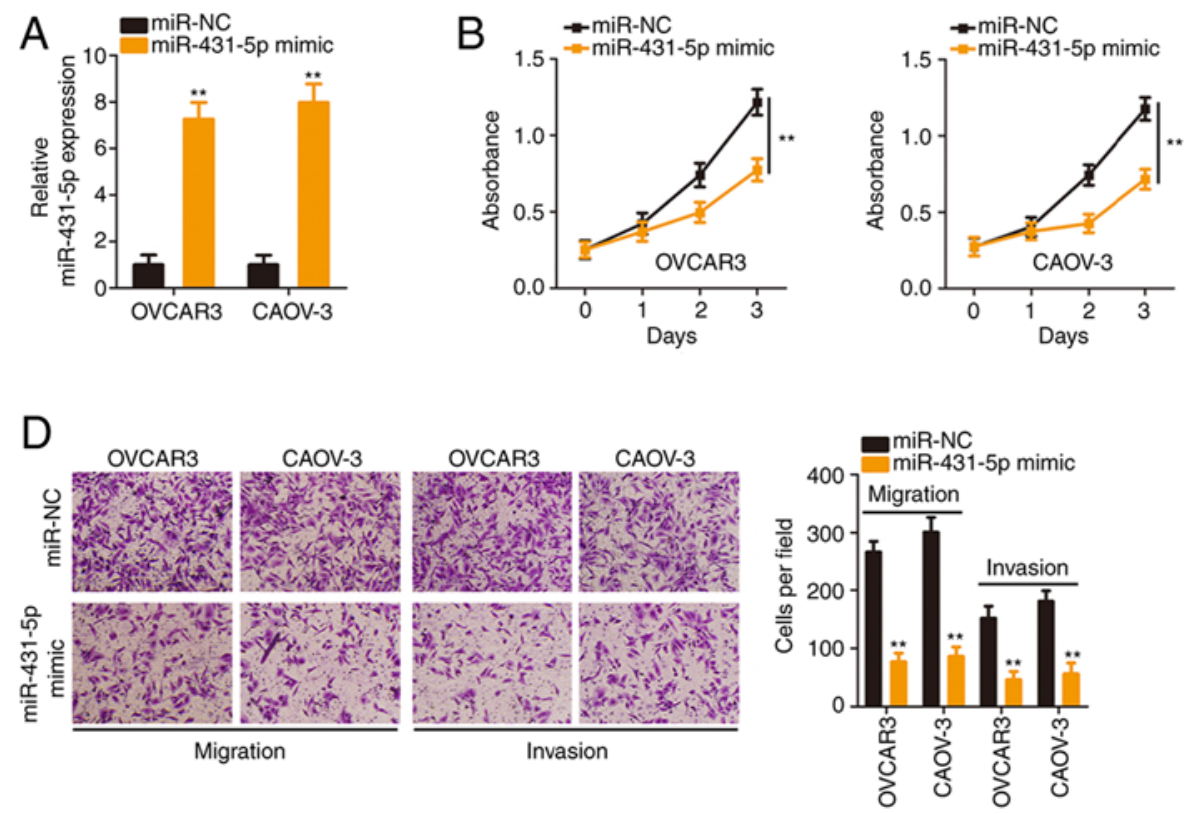

$E$

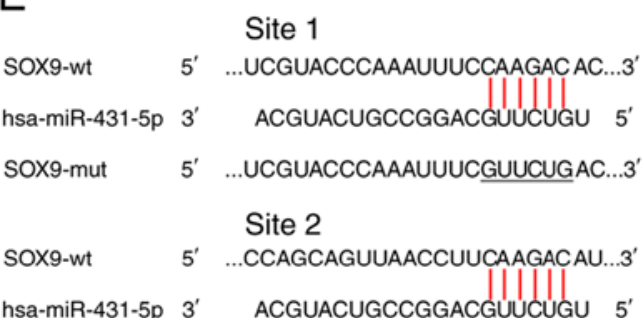

SOX9-mut $\quad 5^{\prime}$...CCAGCAGUUAACCUUGUUCUGAU....3'
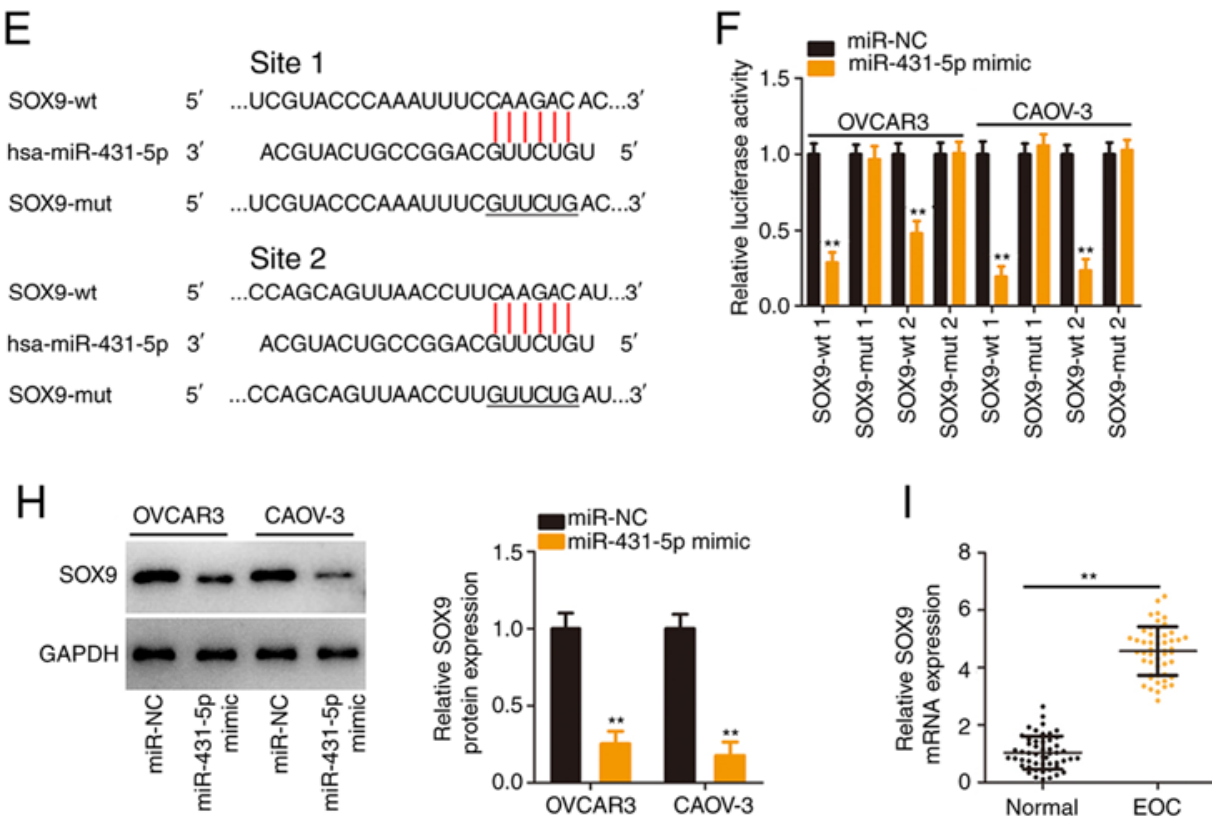
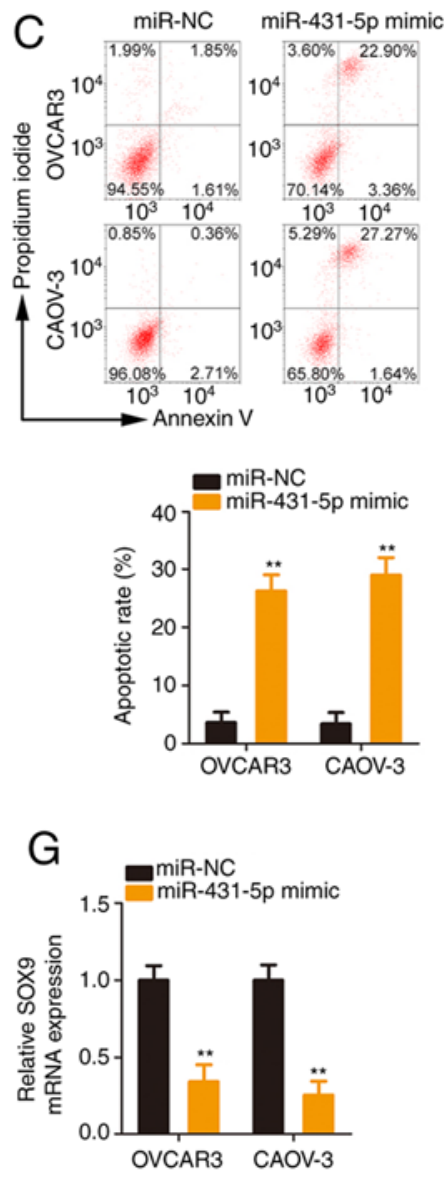

$J$

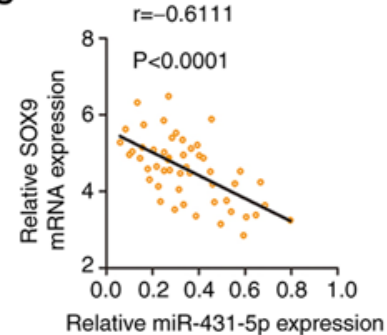

Figure 3. SOX9 is the downstream target of miR-431-5p in EOC cells. (A) The efficiency of miR-431-5p mimic transfection was determined by reverse transcription-quantitative PCR. (B and C) The proliferation and apoptosis of miR-431-5p-overexpressed EOC cells was determined. (D) The migration and invasion abilities were assessed in EOC cells after miR-431-5p upregulation. (E) The 3'-UTR of SOX9 contained two miR-431-5p-binding sites. The mutated sites were also presented. (F) Luciferase activity was analysed in EOC cells which received co-transfection with miR-431-5p mimic or miR-NC and wt or mut reporter plasmids. ( $\mathrm{G}$ and $\mathrm{H}$ ) SOX9 expression was determined in miR-431-5p-overexpressed EOC cells. (I) SOX9 expression was determined in EOC tissues. (J) The relationship between miR-431-5p and SOX9 levels in the 51 EOC tissues. ${ }^{* *} \mathrm{P}<0.01$. SOX9, SRY-box 9; miR, microRNA; EOC, epithelial ovarian cancer; NC, negative control; UTR, untranslated region; wt, wild-type; mut, mutant.

analysis of variance (ANOVA) with Tukey's post hoc test. The overall survival curves were calculated with the Kaplan-Meier method, after which they were analysed with the log-rank test. Pearson's correlation analysis was used to examine the expression correlations. All tests were two-sided, and $\mathrm{P}<0.05$ was considered to indicate a statistically significant difference.

\section{Results}

Silencing of LINC01132 suppresses EOC progression. The present study firstly analysed LINC01132 expression in ovarian cancer tissues through The Cancer Genome Atlas (TCGA) and the Genotype-Tissue Expression (GTEx) databases. LINC01132 was significantly overexpressed in ovarian cancer tissues (Fig. 1A). Next, as compared with adjacent normal tissues, a higher LINC01132 expression level in EOC tissues was confirmed (Fig. 1B). Similarly, LINC01132 was highly expressed in the EOC cell lines (Fig. 1C) compared with OSE cells. Next, all patients in these datasets were classified into either the LINC01132-high expression group or the LINC01132-low expression group on the basis of the median value of LINC01132 in EOC tissues. Patients with EOC 
A

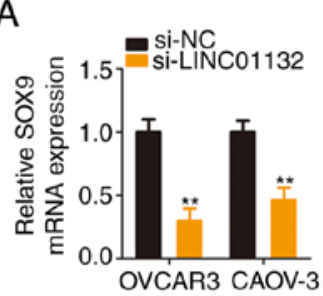

B

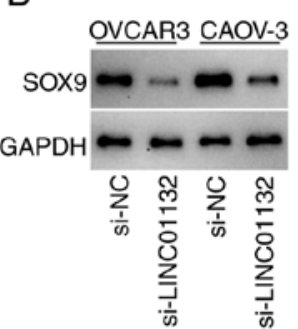

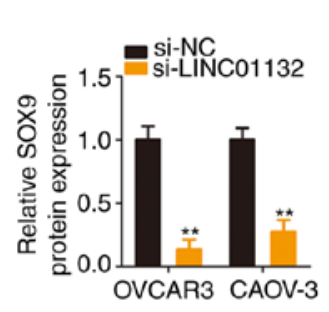

C

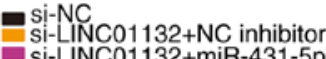
Si-LINC01132+miR-431-5p inhibitor

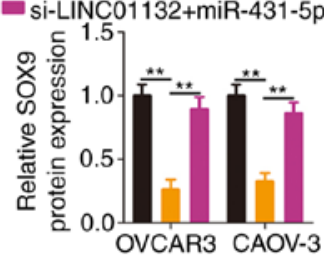
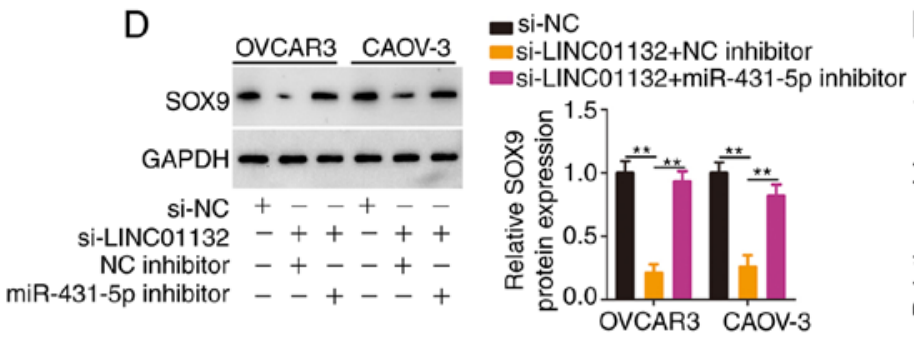

E
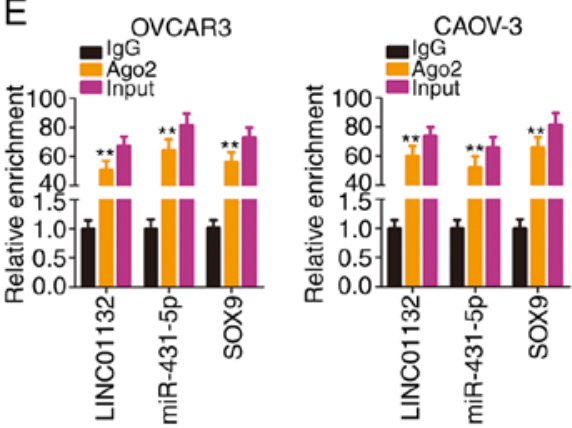

$\mathrm{F}$

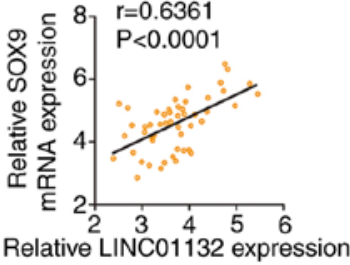

Figure 4. LINC01132 sequesters miR-431-5p and subsequently positively regulates SOX9 expression in EOC. (A and B) SOX9 expression was quantified in EOC cells when LINC01132 was depleted. (C and D) SOX9 levels were detected in EOC cells after co-transfection utilizing si-LINC01132 and miR-431-5p inhibitor or NC inhibitor. (E) The coexistence of LINC01132, miR-431-5p and SOX9 in RNA-induced silencing complexes was verified by RNA immunoprecipitation. (F) The positive expression correlation between LINC01132 and SOX9 in EOC tissues. ${ }^{* *} \mathrm{P}<0.01$. LINC01132, long intergenic non-protein coding RNA 1132; miR, microRNA; SOX9, SRY-box 9; EOC, epithelial ovarian cancer; si-, small interfering; NC, negative control.

characterized by a high LINC01132 expression level exhibited poorer overall survival than those with EOC characterized by a low LINC01132 expression level (Fig. 1D).

Given the upregulation of LINC01132 in EOC, it was questioned whether the dysregulation of LINC01132 was related to the aggressiveness of EOC cells. To this end, the OVCAR3 and CAOV-3 cell lines, which exhibited higher LINC01132 expression than the other EOC cell lines, were selected for subsequent experiments. To avoid off-target effects, si-LINC01132\#1 and si-LINC01132\#2, were separately transfected into OVCAR3 and CAOV-3 cells to effectively induce LINC01132 silencing. RT-qPCR revealed that the 2 siRNAs both exerted satisfactory silencing effects (Fig. 1E). Silencing of LINC01132 significantly inhibited the proliferative ability of EOC cells (Fig. 1F). Additionally, the transfection of si-LINC01132 led to a significant increase in the apoptosis of EOC cells (Fig. 1G). Conversely, cell migratory (Fig. 1H) and invasive (Fig. 1I) capacities were significantly suppressed due to LINC01132 downregulation. Hence, LINC01132 was considered to exert a tumorigenic role in $\mathrm{EOC}$.

LINC01132 sequesters miR-431-5p in EOC. To reveal the mechanisms exerted by LINC01132, the location of LINC01132 was predicted by applying lncLocator. The outcomes revealed that most LINC01132 was distributed in cytoplasm (Fig. 2A), which was subsequently confirmed by applying subcellular fractionation (Fig. 2B). These results indicated that LINC01132 functioned via a ceRNA mechanism. The miRDB tool was utilized to identify the potential target miRNAs of LINC01132. In total, 75 miRNAs contained binding sites for LINC01132. Through TCGA database, a total of 5 miRNAs, including miR-125b-5p, miR-134-3p (Fig. 2C), miR-199a-3p, miR-199b-3p, and miR-431-5p (Fig. 2D), were found to be downregulated in ovarian cancer tissues and thus were selected for experimental verification. Furthermore, the expression of these candidates in EOC cells upon LINC01132 silencing was assessed by RT-qPCR. Only miR-431-5p was overexpressed by si-LINC01132 transfection (Fig. 2E). The influence of LINC01132 on the expression levels of miR-34c-5p was also evaluated and it was demonstrated that LINC01132 failed to affect miR-34c-5p (Fig. 2E). Conversely, miR-431-5p was significantly weakly expressed in EOC tissues (Fig. 2F), and its expression was negatively correlated with LINC01132 (Fig. 2G).

Luciferase reporter assays served to certify the direct binding between miR-431-5p and LINC01132 (Fig. 2H). The upregulation of miR-431-5p significantly decreased the luciferase activity of psiCHECK ${ }^{\mathrm{TM}}$-2-LINC01132-wt (1 and 2), but these regulatory effects on luciferase activity were offset when the binding sites were mutated (1 and 2; Fig. 2I). Furthermore, as demonstrated by the RIP assay, LINC01132 and miR-431-5p were significantly enriched in the Ago2 group (Fig. 2J), indicating the coexistence of two RNAs in the RNA-induced silencing complexes. In summary, LINC01132 sponged miR-431-5p in EOC. 
A

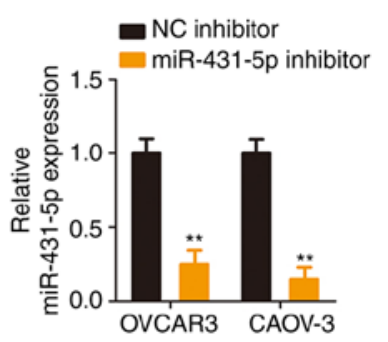

B

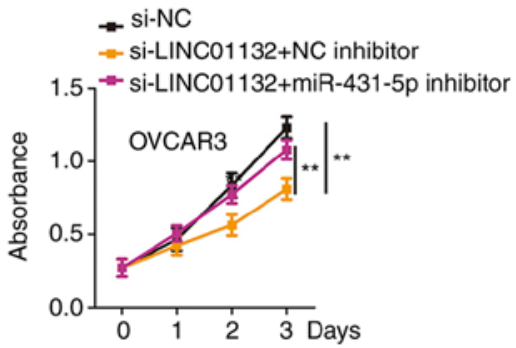

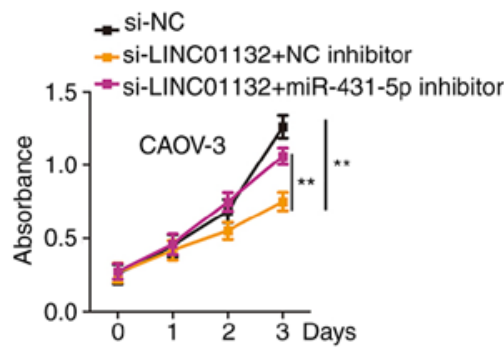
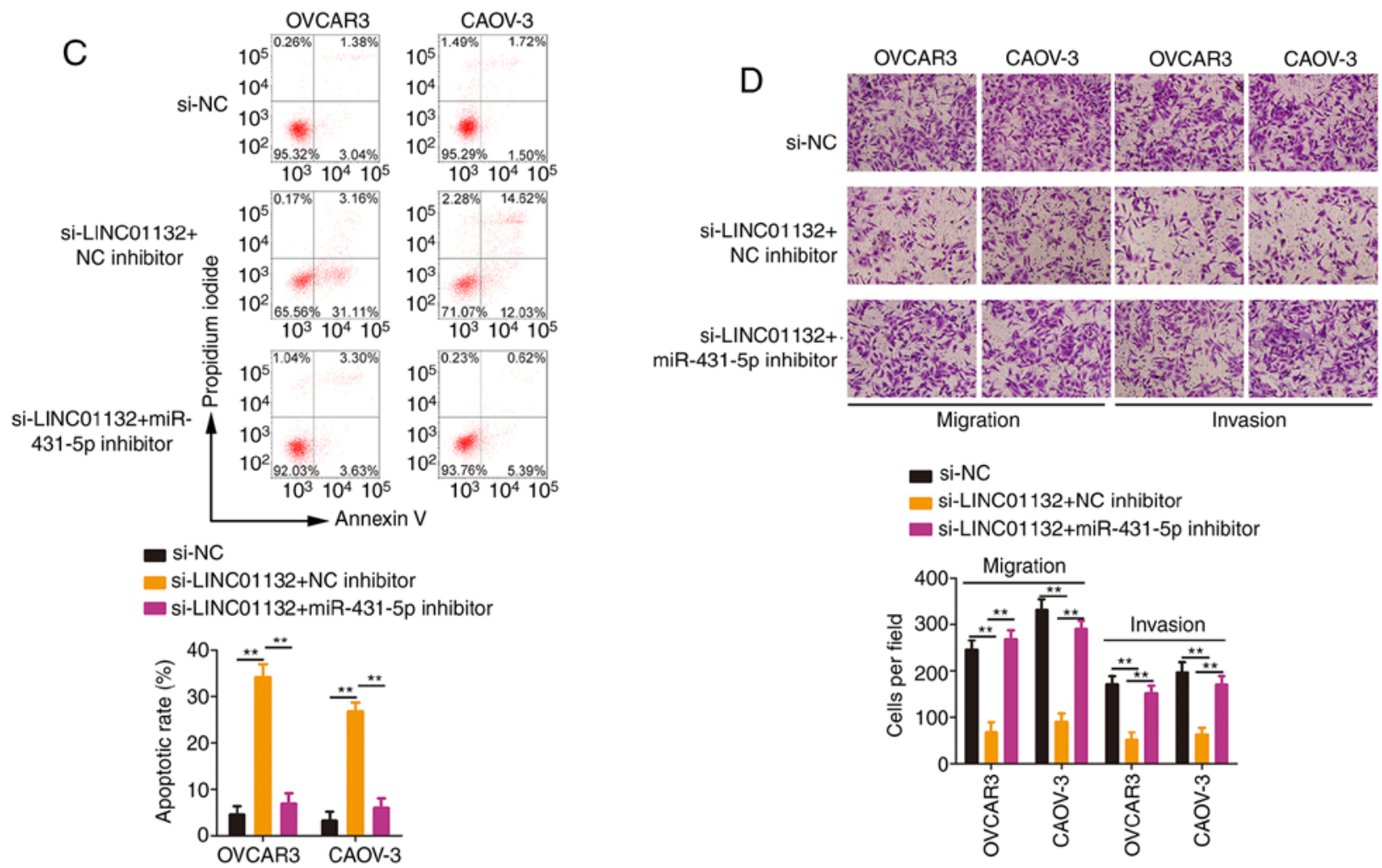

si-LINC01132+miR-431-5p inhibitor

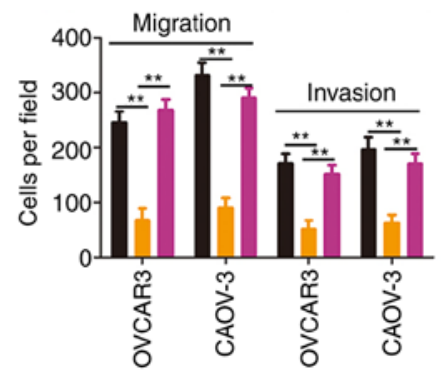

Figure 5. miR-431-5p inhibitor reverses the cancer-inhibiting activities of si-LINC01132 in EOC. (A) The efficiency of miR-431-5p inhibitor transfection was determined by reverse transcription-quantitative PCR. (B-D) LINC01132-deficient EOC cells were treated with miR-431-5p inhibitor or NC inhibitor, followed by the detection of cell proliferation, apoptosis and motility, respectively. ${ }^{* *} \mathrm{P}<0.01$. miR, microRNA; si-, small interfering; LINC01132, long intergenic non-protein coding RNA 1132; EOC, epithelial ovarian cancer; NC, negative control.

LINC01132 regulates SOX9 expression via sequestering $m i R-431-5 p$. The detailed roles of miR-431-5p in EOC were further examined. The overexpression of miR-431-5p in EOC cells by transfection with the miR-431-5p mimic was verified (Fig. 3A). The proliferation of EOC cells was reduced following miR-431-5p overexpression (Fig. 3B). Flow cytometric analysis confirmed that apoptosis was significantly promoted in miR-431-5p-overexpressed EOC cells (Fig. 3C). Additionally, exogenous miR-431-5p expression caused a notable decrease in EOC cell migration and invasion (Fig. 3D).

Bioinformatics analysis was implemented to identify the potential downstream target of miR-431-5p. It was demonstrated that SOX9 contained two binding sites for miR-431-5p (Fig. 3E) and was further studied due to its critical tumour-promoting activities during EOC oncogenesis (24-26). Transfection with miR-431-5p mimic significantly reduced the luciferase activity of psiCHECK ${ }^{\mathrm{TM}}-2$-SOX9-wt (1 and 2) but produced no regulatory effect on the activity of psiCHECK $^{\mathrm{TM}}$-2-SOX9-mut (1 and 2; Fig. 3F). Subsequently, it was revealed that the SOX9 expression (Fig. 3G and H) was downregulated after miR-431-5p mimic transfection. Furthermore, high SOX9 expression in EOC tissues (Fig. 3I) exhibited an inverse relationship with miR-431-5p levels (Fig. 3J). Collectively, these results validated SOX9 as a direct target of miR-431-5p.

Then, it was evaluated whether LINC01132 functioned as a miR-431-5p molecular sponge to regulate SOX9 expression. The absence of LINC01132 significantly reduced SOX9 expression (Fig. 4A and B), whereas this regulation was largely counteracted after miR-431-5p inhibitor treatment (Fig. 4C and D). More importantly, the RIP assay confirmed that LINC01132, miR-431-5p and SOX9 coexisted in RNA-induced silencing complexes (Fig. 4E). In addition, a positive expression relationship between LINC01132 and SOX9 was verified in EOC tissues (Fig. 4F). Collectively, LINC01132 acted as a ceRNA by sequestering miR-431-5p and consequently increasing SOX9 expression.

miR-431-5p/SOX9 axis is required for the carcinogenic actions of LINCO1132 in EOC cells. A series of rescue 

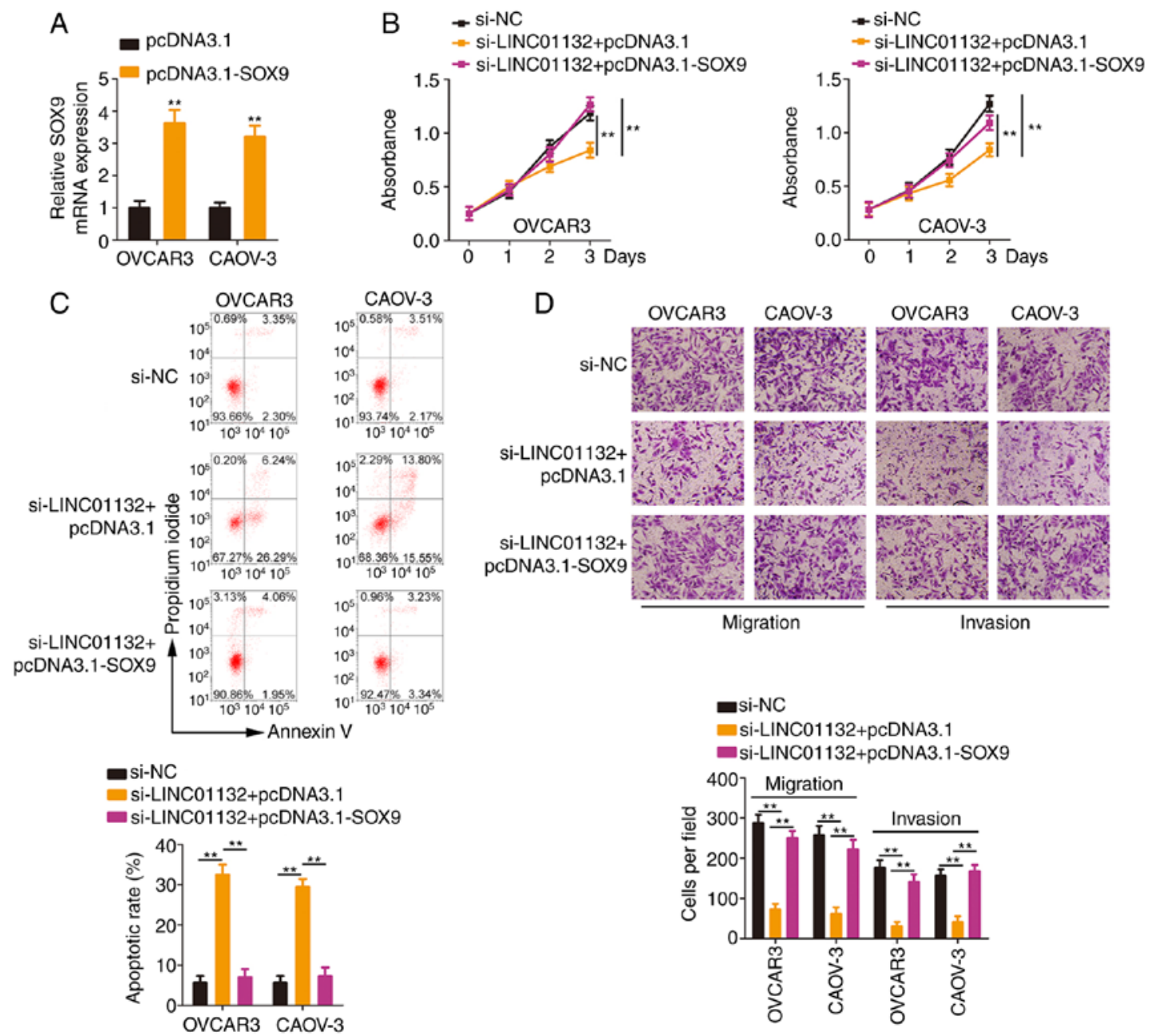

Figure 6. Reintroduction of SOX9 counteracts the effects of si-LINC01132 on EOC cells. (A) The mRNA level of SOX9 in pcDNA3.1-SOX9-transfected EOC cells was determined using reverse transcription-quantitative PCR. (B and C) EOC cells were co-transfected with si-LINC01132 and pcDNA3.1-SOX9 or pcDNA3.1 and then cell proliferation and apoptosis were assessed. (D) The cells aforementioned were also subjected to cell motility determination. ${ }^{* *} \mathrm{P}<0.01$. SOX9, SRY-box 9; si-, small interfering; LINC01132, long intergenic non-protein coding RNA 1132; EOC, epithelial ovarian cancer; NC, negative control.

A

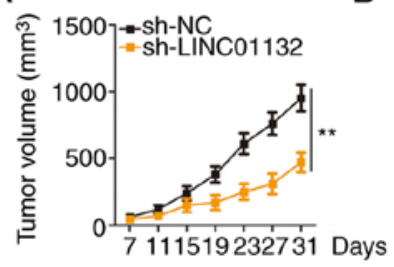

B

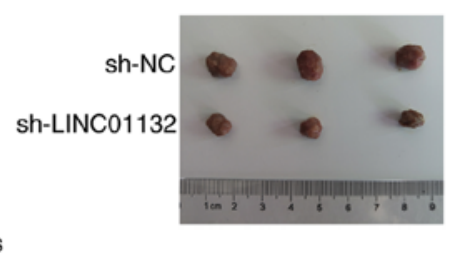

C

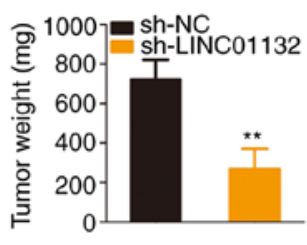

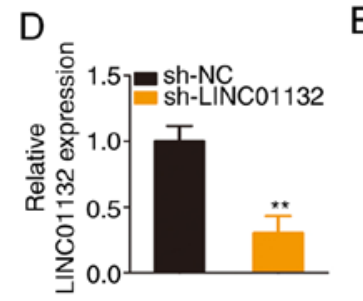

E
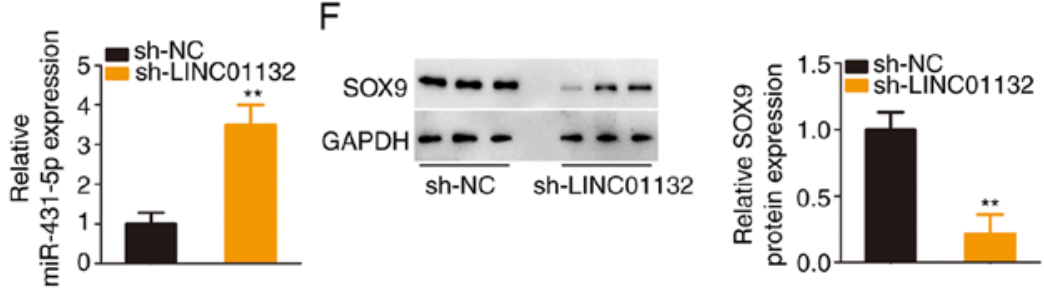

Figure 7. Loss of LINC01132 inhibits tumour growth in vivo. (A) The tumour volumes were monitored at different time-points after tumour cell injection. (B) Representative images demonstrating the tumour xenografts. (C) Weight of the tumour xenografts on the 28th day. (D and E) Expression of LINC01132 and miR-431-5p in tumours. (F) SOX9 protein expression in the tumour xenografts. ${ }^{* *} \mathrm{P}<0.01$. LINC01132, long intergenic non-protein coding RNA 1132; miR, microRNA; SOX9, SRY-box 9; sh-, short hairpin; NC, negative control.

experiments were designed to determine whether modulatory actions of LINC01132 are attributed to the miR-431-5p/SOX9 axis. Transfection of the miR-431-5p inhibitor decreased the expression level of miR-431-5p, suggesting that miR-431-5p 
was successfully silenced in EOC cells (Fig. 5A). The loss of LINC01132 reduced cell proliferation, which was largely restored after miR-431-5p inhibitor treatment (Fig. 5B). Co-transfection with miR-431-5p inhibitor abolished the increase in apoptosis in LINC01132-silenced EOC cells (Fig. 5C). Furthermore, treatment with the miR-431-5p inhibitor recovered the migratory and invasive (Fig. 5D) capacities that were impaired by LINC01132 knockdown.

In addition, the SOX9 overexpression plasmid pcDNA3.1SOX9 (Fig. 6A) was introduced into LINC01132-deficient EOC cells. Re-expression of SOX9 antagonized the cell proliferation suppression and cell apoptosis promotion induced by si-LINC01132 (Fig. 6B and C). Moreover, reduced cell migration and invasion caused by si-LINC01132 was neutralized by overexpressing SOX9 (Fig. 6D). Collectively, the aforementioned results indicated that LINC01132 played tumour-promoting roles in EOC cells via controlling the miR-431-5p/SOX9 axis.

LINC01132 downregulation inhibits xenograft tumour growth in vivo. A tumour xenograft model was implemented to confirm the function of LINC01132 in EOC tumour growth in vivo. Downregulation of LINC01132 significantly slowed tumour growth (Fig. 7A) and reduced tumour size (Fig. 7B). The subcutaneous tumours were harvested at day 31 after injection. It was revealed that the cells transfected with sh-LINC01132 formed tumours with significantly decreased weights (Fig. 7C). Further detection of LINC01132 and miR-431-5p demonstrated that the subcutaneous tumours in the sh-LINC01132 group exhibited a low expression of LINC01132 and a high expression of miR-431-5p (Fig. 7D and E). Furthermore, the LINC01132-downregulated cell-derived tumours exhibited decreased SOX9 expression compared with sh-NC cell-derived tumours (Fig. 7F). Collectively, these results indicated that the depletion of LINC01132 impaired the tumour growth of EOC cells in vivo.

\section{Discussion}

At present, the clinical relationship between lncRNAs and human cancer is a popular research topic in tumour molecular biology given their importance in tumorigenesis and tumour development (27-29). Therefore, IncRNAs are attractive potential therapeutic targets for intervention. As high-throughput sequencing technologies progress, the number of lncRNAs known to be dysregulated in EOC is rapidly increasing (30); however, the detailed contributions of the majority of these IncRNAs remain poorly understood. In the present study, the cellular function and underlying mechanisms of LINC01132 in EOC were revealed in order to provide novel directions and insights for managing EOC.

Multiple studies performed by different scientists have revealed the critical roles of IncRNAs in the oncogenicity of EOC. For instance, NORAD (31), LINC00673 (32) and TC0101441 (33) were upregulated in EOC and have been identified as tumour promoters. Conversely, the low expression of WDFY3-AS2 (34), MAGI2-AS3 (35) and AOC4P (36) in EOC resulted in anti-tumourigenic effects. However, it is unknown whether LINC01132 is involved in EOC malignancy. In the present study, high levels of LINC01132 in EOC were observed in the TCGA database and our own cohort. The overall survival of EOC patients with high LINC01132 levels was significantly lower than patients with low LINC01132 levels. Cell experiments revealed that the knockdown of LINC01132 was capable of inhibiting the proliferation, migration and invasion of EOC cells and promoting apoptosis. The results of the tumour-forming experiment in nude mice were consistent with the in vitro results.

Next, an attempt was made to understand the detailed mechanism by which LINC01132 participates in EOC, which remains mostly uncharacterized. The important roles of lncRNAs in regulating physiological and pathological behaviours are achieved through various mechanisms. The lncRNA-mediated ceRNA theory is a classic mechanism that explains the mechanism of lncRNA action in EOC (12). Cytoplasmic lncRNAs function as endogenous sponges to sequester certain miRNAs and indirectly modulate gene expression by protecting mRNAs from miRNA-induced mRNA dysregulation or translation inhibition (37). Accordingly, IncLocator and subcellular fractionation assays were used to demonstrate that LINC01132 is primary located in the EOC cell cytoplasm. Then, bioinformatics analysis indicated that miR-431-5p may be a target of LINC01132. A luciferase reporter assay verified that LINC01132 possessed a miR-431-5p-binding site.

To explore the ceRNA network mediated by LINC01132 in EOC, the downstream target of miR-431-5p was explored, and the mechanistic analysis identified SOX9 as a target of miR-431-5p. Notably, the data of the present study further revealed that SOX9 was positively controlled by LINC01132 in EOC cells. In rescue experiments, the effects of LINC01132 knockdown on SOX9 expression were abrogated after the inhibition of miR-431-5p. More importantly, LINC01132, miR-431-5p and SOX9 were confirmed to coexist in RNA-induced silencing complexes. Collectively, a new ceRNA network comprising LINC01132, miR-431-5p and SOX9 was identified in EOC.

Differentially expressed miR-431-5p has been validated in several types of human cancer, including EOC (38). Consistent with these studies, the present research confirmed the downregulation of miR-431-5p in EOC and the cancer-inhibiting roles of miR-431-5p in EOC progression. SOX9 was identified as a downstream effector of miR-431-5p in EOC cells. Although the effects of SOX9 in driving EOC initiation and progression have been well elucidated (24-26), the upstream regulatory mechanisms that cause the overexpression of SOX9 in EOC remain largely unknown. Herein, the experimental results revealed a novel LINC01132/miR-431-5p axis that controlled SOX9 expression in EOC cells. Furthermore, miR-431-5p inhibition or SOX9 re-expression eliminated the tumour-suppressive effects of LINC01132 downregulation on the pathological behaviours of EOC cells, demonstrating that SOX9 played critical roles in mediating the LINC01132/miR-431-5p axis-triggered biological functions in EOC.

In the present study, the cell lines CAOV3 and OVCAR3 were used, both of which are highly sensitive to platinum therapy, such as cisplatin or carboplatin (26). The regulation of SOX9 has also been demonstrated to be crucial to cisplatin resistance (26). Additionally, OVCAR8 is a cisplatin-resistant EOC cell line. Thus, the LINC01132/microRNA-431-5p/SOX9 
pathway may perform crucial roles in the regulation of cisplatin sensitivity in EOC cells. The present study did not evaluate this effect, which is a limitation that will be addressed in the near future.

In summary, the present study was the first to report, to the best of our knowledge, that LINC01132 was upregulated in EOC and exhibited a significant relationship with poor clinical prognosis. LINC01132 was revealed to exert oncogenic effects in EOC cells by controlling the outcome of the $\mathrm{miR}-431-5 \mathrm{p} / \mathrm{SOX} 9$ axis. LINC01132/miR-431-5p/SOX9 is anticipated to be an attractive target for the treatment and prognostic evaluation of EOC.

\section{Acknowledgements}

Not applicable.

\section{Funding}

No funding was received.

\section{Availability of data and materials}

All datasets generated and analysed during the present study are available from the corresponding author on reasonable request.

\section{Authors' contributions}

JC and WZ devised the current research. All the experiments were performed by WZ, XX and JC. JC and WZ analysed the obtained data. The manuscript was drafted by JC and WZ. All authors read and approved the final manuscript.

\section{Ethics approval and consent to participate}

The collection and use of human tissues was approved by the Ethics Committee of Weifang People's Hospital (Weifang, China). All experimental steps involving animals were conducted with approval from the Institutional Animal Care and Use Committee of Weifang People's Hospital.

\section{Patient consent for publication}

Not applicable.

\section{Competing interests}

The authors declare that they have no competing interests.

\section{References}

1. Bray F, Ferlay J, Soerjomataram I, Siegel RL, Torre LA and Jemal A: Global cancer statistics 2018: GLOBOCAN estimates of incidence and mortality worldwide for 36 cancers in 185 countries. CA Cancer J Clin 68: 394-424, 2018.

2. AllemaniC,MatsudaT,DiCarlo V,HarewoodR,MatzM,NikšićM, Bonaventure A, Valkov M, Johnson CJ, Estève J, et al: Global surveillance of trends in cancer survival 2000-14 (CONCORD-3) Analysis of individual records for 37,513,025 patients diagnosed with one of 18 cancers from 322 population-based registries in 71 countries. Lancet 391: 1023-1075, 2018.
3. Di Lorenzo G, Ricci G, Severini GM, Romano F and Biffi S: Imaging and therapy of ovarian cancer: Clinical application of nanoparticles and future perspectives. Theranostics 8: 4279-4294, 2018.

4. Kuroki L and Guntupalli SR: Treatment of epithelial ovarian cancer. BMJ 371: m3773, 2020.

5. Gourley $\mathrm{C}$ and Bookman MA: Evolving concepts in the management of newly diagnosed epithelial ovarian cancer. J Clin Oncol 37: 2386-2397, 2019.

6. Rojas V, Hirshfield KM, Ganesan S and Rodriguez-Rodriguez L: Molecular characterization of epithelial ovarian cancer: Implications for diagnosis and treatment. Int J Mol Sci 17: 2113, 2016.

7. Sundar S, Neal RD and Kehoe S: Diagnosis of ovarian cancer. BMJ 351: h4443, 2015.

8. Nagano $T$ and Fraser P: No-nonsense functions for long noncoding RNAs. Cell 145: 178-181, 2011.

9. Qiu L, Tang Q, Li G and Chen K: Long non-coding RNAs as biomarkers and therapeutic targets: Recent insights into hepatocellular carcinoma. Life Sci 191: 273-282, 2017.

10. Wang Y, Zhang M and Zhou F: Biological functions and clinical applications of exosomal long non-coding RNAs in cancer. J Cell Mol Med 24: 11656-11666, 2020.

11. Pei C, Gong X and Zhang Y: LncRNA MALAT-1 promotes growth and metastasis of epithelial ovarian cancer via sponging microrna-22. Am J Transl Res 12: 6977-6987, 2020.

12. Braga EA, Fridman MV, Moscovtsev AA, Filippova EA, Dmitriev AA and Kushlinskii NE: LncRNAs in ovarian cancer progression, metastasis, and main pathways: ceRNA and alternative mechanisms. Int J Mol Sci 21: 8855, 2020.

13. Wambecke A, Ahmad M, Lambert B, Joly F, Poulain L, Denoyelle $\mathrm{C}$ and Meryet-Figuiere M: The influence of long non-coding RNAs on the response to chemotherapy in ovarian cancer. Gynecol Oncol 156: 726-733, 2020.

14. Zeng S, Liu S, Feng J, Gao J and Xue F: Upregulation of IncRNA AB073614 functions as a predictor of epithelial ovarian cancer prognosis and promotes tumor growth in vitro and in vivo. Cancer Biomark 24: 421-428, 2019.

15. Wu W, Gao H, Li X, Zhu Y, Peng S, Yu J, Zhan G, Wang J, Liu N and Guo X: LncRNA TPT1-AS1 promotes tumorigenesis and metastasis in epithelial ovarian cancer by inducing TPT1 expression. Cancer Sci 110: 1587-1598, 2019.

16. Zhao J and Liu HR: Down-regulation of long noncoding RNA DLX6-AS1 defines good prognosis and inhibits proliferation and metastasis in human epithelial ovarian cancer cells via Notch signaling pathway. Eur Rev Med Pharmacol Sci 23: 3243-3252, 2019.

17. Lu TX and Rothenberg ME: MicroRNA. J Allergy Clin Immunol 141: 1202-1207, 2018.

18. Nguyen VHL, Yue C, Du KY, Salem M, O'Brien J and Peng C: The role of microRNAs in epithelial ovarian cancer metastasis. Int J Mol Sci 21: 7093, 2020.

19. Staicu CE, Predescu DV, Rusu CM, Radu BM, Cretoiu D, Suciu N, Crețoiu SM and Voinea SC: Role of microRNAs as clinical cancer biomarkers for ovarian cancer: A short overview. Cells 9: 169, 2020

20. Khan S, Ayub H, Khan T and Wahid F: MicroRNA biogenesis, gene silencing mechanisms and role in breast, ovarian and prostate cancer. Biochimie 167: 12-24, 2019.

21. Niu ZS, Wang WH, Dong XN and Tian LM: Role of long noncoding RNA-mediated competing endogenous RNA regulatory network in hepatocellular carcinoma. World $\mathrm{J}$ Gastroenterol 26: 4240-4260, 2020.

22. Weng W, Zhang Z, Huang W, Xu X, Wu B, Ye T, Shan Y, Shi K and Lin Z: Identification of a competing endogenous RNA network associated with prognosis of pancreatic adenocarcinoma. Cancer Cell Int 20: 231, 2020.

23. Livak KJ and Schmittgen TD: Analysis of relative gene expression data using real-time quantitative PCR and the 2(-Delta Delta C(T)) method. Methods 25: 402-408, 2001

24. Raspaglio G,Petrillo M,Martinelli E,Li Puma DD, Mariani M,De Donato M, Filippetti F, Mozzetti S, Prislei S, Zannoni GF, et al: Sox9 and Hif- $2 \alpha$ regulate TUBB3 gene expression and affect ovarian cancer aggressiveness. Gene 542: 173-181, 2014.

25. Malki S, Bibeau F, Notarnicola C, Roques S, Berta P, Poulat F and Boizet-Bonhoure B: Expression and biological role of the prostaglandin D synthase/SOX9 pathway in human ovarian cancer cells. Cancer Lett 255: 182-193, 2007.

26. Xiao S, Li Y, Pan Q, Ye M, He S, Tian Q and Xue M: MiR-34c/SOX9 axis regulates the chemoresistance of ovarian cancer cell to cisplatin-based chemotherapy. J Cell Biochem 120: 2940-2953, 2019. 
27. McCabe EM and Rasmussen TP: lncRNA involvement in cancer stem cell function and epithelial-mesenchymal transitions. Semin Cancer Biol: Dec 17, 2020 (Epub ahead of print).

28. Qian Y, Shi L and Luo Z: Long non-coding RNAs in cancer: Implications for diagnosis, prognosis, and therapy. Front Med (Lausanne) 7: 612393, 2020.

29. Chen S and Shen X: Long noncoding RNAs: Functions and mechanisms in colon cancer. Mol Cancer 19: 167, 2020

30. Nikpayam E, Tasharrofi B, Sarrafzadeh S and Ghafouri-Fard S: The role of long non-coding RNAs in ovarian cancer. Iran Biomed J 21: 3-15, 2017.

31. Tong L, Ao Y, Zhang H, Wang K, Wang Y and Ma Q: Long noncoding RNA NORAD is upregulated in epithelial ovarian cancer and its downregulation suppressed cancer cell functions by competing with miR-155-5p. Cancer Med 8: 4782-4791, 2019.

32. Zheng T, Qiu J, Li C, Lin X, Tang X and Hua K: Long noncoding RNA LINC00673 promotes the proliferation and metastasis of epithelial ovarian cancer by associating with opioid growth factor receptor. Onco Targets Ther 12: 6145-6156, 2019.

33. Qiu JJ, Lin XJ, Tang XY, Zheng TT, Zhang XY and Hua KQ Long noncoding RNA TC0101441 induces epithelial-mesenchymal transition in epithelial ovarian cancer metastasis by downregulating KiSS1. Int J Cancer 146: 2588-2598, 2020.
34. Li W, Ma S, Bai X, Pan W, Ai L and Tan W: Long noncoding RNA WDFY3-AS2 suppresses tumor progression by acting as a competing endogenous RNA of microRNA-18a in ovarian cancer. J Cell Physiol 235: 1141-1154, 2020.

35. Gokulnath P, de Cristofaro T, Manipur I, Di Palma T, Soriano AA, Guarracino MR and Zannini M: Long non-coding RNA MAGI2-AS3 is a new player with a tumor suppressive role in high grade serous ovarian carcinoma. Cancers (Basel) 11: 2008, 2019

36. Lin X, Tang X, Zheng T, Qiu J and Hua K: Long non-coding RNA AOC4P suppresses epithelial ovarian cancer metastasis by regulating epithelial-mesenchymal transition. J Ovarian Res 13: $45,2020$.

37. Qu J, Li M, Zhong $\mathrm{W}$ and $\mathrm{Hu} \mathrm{C}$ : Competing endogenous RNA in cancer: A new pattern of gene expression regulation. Int J Clin Exp Med 8: 17110-17116, 2015.

38. Yang L, Lv Q, Liu J, Qi S and Fu D: miR-431 regulates granulosa cell function through the IRS2/PI3K/AKT signaling pathway. J Reprod Dev 66: 231-239, 2020. 